\title{
SERGIU RUdEANU
}

\section{Programmation bivalente à plusieurs fonctions économiques}

Revue française d'automatique, d'informatique et de recherche opérationnelle. Recherche opérationnelle, tome 3, n V2 (1969), p. 13-29.

<http://www.numdam.org/item?id=RO_1969_3_2_13_0>

(C) AFCET, 1969, tous droits réservés.

L'accès aux archives de la revue « Revue française d'automatique, d'informatique et de recherche opérationnelle. Recherche opérationnelle » implique l'accord avec les conditions générales d'utilisation (http://www.numdam.org/ legal.php). Toute utilisation commerciale ou impression systématique est constitutive d'une infraction pénale. Toute copie ou impression de ce fichier doit contenir la présente mention de copyright.

\section{Numdam}

Article numérisé dans le cadre du programme

Numérisation de documents anciens mathématiques

http://www.numdam.org/ 
R.I.R.O.

(3 année, No V-2, 1969, p. 13-30)

\title{
PROGRAMMATION BIVALENTE A PLUSIEURS FONCTIONS EGONOMIQUES
}

\author{
par Sergiu Rudeanu (2)
}

Résumé. - Le succès de beaucoup d'activités pratiques peut être mesuré par rapport $\grave{a}$ plusieurs critères d'efficacité (par exemple, dans un problème de transport on peut envisager le coût, la vitesse, la quantité de marchandise transportée, etc.). Nous sommes ainsi conduits aux problèmes de programmation mathématique à plusieurs fonctions économiques qui ne peuvent pas être optimisées simultanément. Pour résoudre de tels problèmes, on peut envisager trois approches :

10 Remplacer les diverses fonctions économiques par une seule fonction.

$2^{\circ}$ Etablir un ordre préférentiel parmi les fonctions économiques.

$3^{0}$ Optimiser le vecteur des fonctions économiques par rapport à l'ordre défini composante par composante (qui est seulement un ordre partiel, de sorte que plusieurs optima incomparables peuvent exister).

Ce travail étudie les approches $2^{\circ}$ et $3^{\circ}$ dans le. cas de la programmation bivalente $(0,1)$.

\section{$\S 0$. INTRODUCTION}

Les problèmes de la recherche opérationnelle sont énoncés d'habitude sous la forme suivante : optimiser une fonction dite "économique ", dont les variables sont sujettes à certaines restrictions. La fonction économique représente un critère selon lequel on apprécie le succès d'une certaine activité. Il convient cependant de ne pas oublier que souvent il y a plusieurs critères dont il faudrait tenir compte ; ces critères sont contradictoires en ce sens que les fonctions économiques qui leur correspondent ne peuvent être optimisées simultanément. Ainsi, par exemple, dans un problème de transport on peut envisager le coût, la rapidité, ou la quantité de marchandise transportée ; l'activité d'une usine peut être appréciée selon la quantité du produit qu'elle fabrique, ou bien selon le coût de ce produit ; etc. Adopter un critère c'est négliger les autres, ce qui ne va pas

(1) Communication présentée à la Session scientifique annuelle du Centre de calcul économique et cybernétique économique. Bucarest, 21-22 février 1969.

(2) Faculté de Mathématique, Université de Bucarest. 
sans inconvénients dans certains cas. Voilà pourquoi certains auteurs ont abordé des problèmes d'optimisation selon plusieurs critères à la fois [2], [5] ; signalons en particulier la méthode "Electre » [7], qui a commencé à être appliquée en France [4] et en Roumanie [1] (1).

Dans son travail [2], P. Bod aborde ce sujet en proposant trois points de vue possibles, que nous allons reprendre ci-dessous $(\S 1)$ pour traiter ensuite un cas qui exige des méthodes spéciales : celui de la programmation à variables bivalentes $(0,1)(\S 2)$. Des exemples sont donnés au paragraphe 3 . Nous remarquons finalement que la méthode "Electre " semble très prometteuse pour la continuation du présent travail $(\$ 4)$.

\section{§1. POSITION DU PRobleme}

Posons d'abord le problème de façon générale, c'est-à-dire sans préciser le domaine des variables. Considérons donc un système quelconque de contraintes, que nous désignerons par

$$
C\left(x_{1}, \ldots, x_{n}\right),
$$

et $m$ fonctions économiques à valeurs réelles

$$
f_{1}\left(x_{1}, \ldots, x_{n}\right), \ldots, f_{m}\left(x_{1}, \ldots, x_{n}\right) ;
$$

sans restreindre la généralité, nous pouvons supposer que ces fonctions sont à maximiser. Comme il n'existe, en général, aucun point $\left(x_{1}^{*}, \ldots, x_{n}^{*}\right)$ qui maximise à la fois les $m$ fonctions sous les restrictions données, nous sommes obligés d'adopter l'un des trois points de vue ci-dessous.

$1^{\circ}$ Remplacer les $m$ fonctions par une seule fonction économique à valeurs réelles

$$
f\left(x_{1}, \ldots, x_{n}\right)=g\left(f_{1}\left(x_{1}, \ldots, x_{n}\right), \ldots, f_{m}\left(x_{1}, \ldots, x_{n}\right)\right),
$$

où $g\left(y_{1}, \ldots, y_{m}\right)$ est une fonction non-décroissante en chacune des variables $y_{1}, \ldots, y_{m}$; maximiser $f\left(x_{1}, \ldots, x_{n}\right)$ sous les contraintes $C\left(x_{1}, \ldots, x_{n}\right)$. Plusieurs exemples de fonctions $g$ sont donnés par A. M. Geoffrion [5]; un bon candidat semble être la fonction $g\left(y_{1}, \ldots, y_{m}\right):=\sum_{i=1}^{m} \rho_{i} y_{i}$, avec des poids $\rho_{i}>0(i=1, \ldots, m)$. Il nous semble que le choix de la fonction $g$ - et en particulier le choix des poids $p_{i}$ - est un problème qui relève de la nature particulière du programme; voir [3].

$2^{\circ}$ Établir un ordre préférentiel des fonctions économiques, soit $f_{1}, f_{2}, \ldots, f_{m}$. Dans ce cas-ci, résoudre le programme c'est déterminer d'abord tous les points $\left(x_{1}, \ldots, x_{n}\right)$ où la fonction $f_{1}$ atteint son maximum conditionné par (1); s'il y en a plusieurs, il faut garder seulement le(s) point(s) qui maximise(nt) la fonction $f_{2}$; etc.

(1) Il est bien possible que d'autres travaux abordant le même sujet aient échappé à notre attention. 
$\mathrm{Du}$ point de vue algébrique, cela revient à remplacer les $m$ fonctions économiques par une seule fonction économique vectorielle, à savoir

$$
F\left(x_{1}, \ldots, x_{n}\right)=\left(f_{1}\left(x_{1}, \ldots, x_{n}\right), \ldots, f_{m}\left(x_{1}, \ldots, x_{n}\right)\right),
$$

dont les valeurs appartiennent au produit cartésien,

$$
R^{m}=\left\{\left(y_{1}, \ldots, y_{m}\right) \mid y_{1}, \ldots, y_{m} \text { réels }\right\},
$$

muni de l'ordre lexicographique. Nous rappelons la définition de cette relation (1):

$$
\begin{aligned}
\left(y_{1}^{\prime}, \ldots, y_{m}^{\prime}\right) \leqslant\left(y_{1}^{\prime \prime}, \ldots, y_{m}^{\prime \prime}\right) & \Leftrightarrow\left(y_{1}^{\prime}<y_{1}^{\prime \prime}\right) \vee\left(y_{1}^{\prime}=y_{1}^{\prime \prime} \& y_{2}^{\prime}<y_{2}^{\prime \prime}\right) \\
\ldots & \vee\left(y_{1}^{\prime}=y_{1}^{\prime \prime} \& \ldots \& y_{m-1}^{\prime}=y_{m-1}^{\prime \prime} \& y_{m}^{\prime}<y_{m}^{\prime \prime}\right) \\
& \vee\left(y_{1}^{\prime}=y_{1}^{\prime \prime} \& \ldots \& y_{m}^{\prime}=y_{m}^{\prime \prime}\right) .
\end{aligned}
$$

Si l'on se rappelle aussi la définition de l'égalité des vecteurs, à savoir

$$
\left(y_{1}^{\prime}, \ldots, y_{m}^{\prime}\right)=\left(y_{1}^{\prime \prime}, \ldots, y_{m}^{\prime \prime}\right) \Leftrightarrow y_{1}^{\prime}=y_{1}^{\prime \prime} \& \ldots \& y_{m}^{\prime}=y_{m}^{\prime \prime},
$$

on vérifie immédiatement que la relation (6) est bien une relation d'ordre, c'est-à-dire

$$
\begin{aligned}
& \left(y_{1}^{\prime}, \ldots, y_{m}^{\prime}\right)=\left(y_{1}^{\prime \prime}, \ldots, y_{m}^{\prime \prime}\right) \Leftrightarrow\left(y_{1}^{\prime}, \ldots, y_{m}^{\prime}\right) \leqslant\left(y_{1}^{\prime \prime}, \ldots, y_{m}^{\prime \prime}\right) \\
& \&\left(y_{1}^{\prime \prime}, \ldots, y_{m}^{\prime \prime}\right) \leqslant\left(y_{1}^{\prime}, \ldots, y_{m}^{\prime}\right), \\
& \text { (9) }\left(y_{1}^{\prime}, \ldots, y_{m}^{\prime}\right) \leqslant\left(y_{1}^{\prime \prime}, \ldots, y_{m}^{\prime \prime}\right) \&\left(y_{1}^{\prime \prime}, \ldots, y_{m}^{\prime \prime}\right) \leqslant\left(y_{1}^{\prime \prime \prime}, \ldots, y_{m}^{\prime \prime \prime}\right) \\
& \Rightarrow\left(y_{1}^{\prime}, \ldots, y_{m}^{\prime}\right) \leqslant\left(y_{1}^{\prime \prime \prime}, \ldots, y_{m}^{\prime \prime \prime}\right) ;
\end{aligned}
$$

de plus, l'ordre est total, c'est-à-dire que pour toute paire de vecteurs $\left(y_{1}^{\prime}, \ldots, y_{m}^{\prime}\right),\left(y_{1}^{\prime \prime}, \ldots, y_{m}^{\prime \prime}\right)$, on a

$$
\left(y_{1}^{\prime}, \ldots, y_{m}^{\prime}\right) \leqslant\left(y_{1}^{\prime \prime}, \ldots, y_{m}^{\prime \prime}\right) \vee\left(y_{1}^{\prime \prime}, \ldots, y_{m}^{\prime \prime}\right) \leqslant\left(y_{1}^{\prime}, \ldots, y_{m}^{\prime}\right) \text {. }
$$

L'existence d'une ou de plusieurs solutions du programme $2^{\circ}$ est assurée si, par exemple, nous supposons que l'ensemble des solutions du système (1) est fini et non vide ; ou bien, plus généralement, si l'ensemble des points où $f_{1}$ atteint son maximum conditionné par (1), est fini et non vide.

$3^{0}$ Maximiser le vecteur des fonctions économiques, composante par composante. Plus précisément, cette troisième approche veut dire ceci. On considère le produit cartésien $R^{m}$ muni de l'ordre partiel défini composante par composante, c'est-à-dire

$$
\left(y_{1}^{\prime}, \ldots, y_{m}^{\prime}\right) \leqslant\left(y_{1}^{\prime \prime}, \ldots, y_{m}^{\prime \prime}\right) \Leftrightarrow y_{1}^{\prime} \leqslant y_{1}^{\prime \prime} \& \ldots \& y_{m}^{\prime} \leqslant y_{m}^{\prime \prime} .
$$

(1) Les signes logiques $\&, V, \Rightarrow$, $\Leftrightarrow$, ont leur sens habituel. 
On vérifie immédiatement que cette relation jouit bien des propriétés (8) et (9) d'une relation d'ordre, sans toutefois satisfaire à la condition (10); autrement dit, il y a des paires de vecteurs

$$
\left(y_{1}^{\prime}, \ldots, y_{m}^{\prime}\right),\left(y_{1}^{\prime \prime}, \ldots, y_{m}^{\prime \prime}\right)
$$

incomparables, c'est-à-dire tels que

$$
\left(y_{1}^{\prime}, \ldots, y_{m}^{\prime}\right) \leqslant\left(y_{1}^{\prime \prime}, \ldots, y_{m}^{\prime \prime}\right) \text { et }\left(y_{1}^{\prime \prime}, \ldots, y_{m}^{\prime \prime}\right) \leqslant\left(y_{1}^{\prime}, \ldots, y_{m}^{\prime}\right) \text {. }
$$

Considérons l'ense mble $C$ formé par tous les points $\left(x_{1}, \ldots, x_{n}\right)$ qui satisfont aux contraintes (1):

$$
C=\left\{\left(x_{1}, \ldots, x_{n}\right) \mid C\left(x_{1}, \ldots, x_{n}\right)\right\}
$$

et soit $F(C)$ l'ensemble des valeurs de la restriction $F \mid C$ de la fonction (4) au domaine $C$ :

$$
F(C)=\left\{F\left(x_{1}, \ldots, x_{n}\right) \mid\left(x_{1}, \ldots, x_{n}\right) \in C\right\} \subseteq R^{m}
$$

Supposons les contraintes (1) compatibles, c'est-à-dire $C \neq \varnothing$ ou bien, ce qui revient au même, $F(C) \neq \varnothing$. L'ordre (11) n'étant pas total, l'ensemble $F(C)$ n'aura pas, en général, un plus grand élément, qui est nécessairement unique, mais, sous des hypothèses adéquates, plusieurs éléments maximaux (1). Rappelons qu'un élément maximal $\left(y_{1}^{*}, \ldots, y_{m}^{*}\right)$ de $F(C)$ est défini par les deux propriétés suivantes :

$$
\begin{aligned}
& \left(y_{1}^{*}, \ldots, y_{m}^{*}\right) \in F(C) \\
& \left(y_{1}, \ldots, y_{m}\right) \in F(C) \&\left(y_{1}^{*}, \ldots, y_{m}^{*}\right) \leqslant\left(y_{1}, \ldots, y_{m}\right) \\
& \quad \Rightarrow\left(y_{1}^{*}, \ldots, y_{m}^{*}\right)=\left(y_{1}, \ldots, y_{m}\right) .
\end{aligned}
$$

Nous dirons aussi qu'un élément maximal de l'ensemble $F(C)$ est une valeur maximale de la fonction $F \mid C$. Le plus grand élément (élément maximum) de $F(C)$ satisfait (14.1) et

$$
\left(y_{1}, \ldots, y_{m}\right) \in F(C) \Rightarrow\left(y_{1}, \ldots, y_{m}\right) \leqslant\left(y_{1}^{*}, \ldots, y_{m}^{*}\right) \text {. }
$$

Le plus grand élément, s'il existe, est le seul élément maximal de $F(C)$.

Le problème qu'on se pose tout naturellement est de savoir si l'ensemble $F(C)$ admet des éléments maximaux et dans l'affirmative, trouver un vecteur

$$
\left(x_{1}^{*}, \ldots, x_{n}^{*}\right) \in C
$$

tel que

$$
F\left(x_{1}^{*}, \ldots, x_{n}^{*}\right)=\left(y_{i}^{*}, \ldots, y_{m}^{*}\right)
$$

soit un élément maximal de $F(C)$.

(1) Dans certains cas particuliers il peut arriver que $F(C)$ ait un plus grand élément; voir paragraphe 3 , exemple 2 . 
Or, on voit immédiatement que toute solution du problème $2^{\circ}$ ci-dessus, pour un ordre quelconque des fonctions économiques, est aussi une solution du problème que nous venons de poser. Nous verrons $(\$ 3$, exemple 1) que la réciproque n'est pas vraie.

On peut aussi se poser le problème suivant: trouver une valeur maximale de $F \mid C$ qui améliore une valeur donnée. Autrement dit, on nous donne une solution particulière $\left(x_{1}^{\prime}, \ldots, x_{n}^{\prime}\right)$ du système des contraintes et nous voulons déterminer une valeur maximale (16) de $F \mid C$ telle que

$$
F\left(x_{1}^{\prime}, \ldots, x_{n}^{\prime}\right) \leqslant F\left(x_{1}^{*}, \ldots, x_{n}^{*}\right) .
$$

A cet effet, il suffit d'ajouter aux contraintes les conditions supplémentaires

$$
f_{i}\left(x_{1}, \ldots, x_{n}\right) \geqslant y_{i}^{\prime} \quad(i=1, \ldots, m),
$$

où les constantes $y_{i}^{\prime}$ sont

$$
y_{i}^{\prime}=f_{i}\left(x_{1}^{\prime}, \ldots, x_{n}^{\prime}\right) \quad(i=1, \ldots, m),
$$

le problème étant ainsi ramené au cas précédent.

Si l'on veut déterminer plusieurs (tous les) points $\left(x_{1}^{*}, \ldots, x_{n}^{*}\right)$ où la fonction $F\left(x_{1}, \ldots, x_{n}\right)$ atteint une certaine valeur maximale $\left(y_{1}^{*}, \ldots, y_{m}^{*}\right)$, il faut trouver plusieurs (toutes les) solutions du système $\{(1),(20)\}$ suivant :

$$
\begin{aligned}
& C\left(x_{1}, \ldots, x_{n}\right) \\
& f_{i}\left(x_{1}, \ldots, x_{n}\right)=y_{i}^{*} \quad(i=1, \ldots, m) .
\end{aligned}
$$

Il peut arriver qu'un vecteur maximal $\left(y_{1}^{*}, \ldots, y_{m}^{*}\right)$ de $F(C)$ soit inacceptable parce que certaines des composantes $y_{i}$ ont des valeurs trop petites. Pour éviter cet inconvénient, il suffit d'ajouter au système (1) des contraintes de la forme

$$
f_{i}\left(x_{1}, \ldots, x_{n}\right) \geqslant m_{i} \quad(i=1, \ldots, m),
$$

où les $m_{i}$ sont des limites inférieures résultant des particularités du problème étudié.

Parmi les trois points de vue ci-dessus, l'approche $3^{\circ}$ nous semble la plus appropriée à la nature du problème posé, pourvu, bien entendu, que les moyens techniques nous permettent d'effectuer les opérations indiquées. Nous allons voir que tel est le cas de la programmation bivalente, où l'on peut même espérer de résoudre le problème général de trouver tous les vecteurs maximaux de $F(C)$ et tous les points où la fonction $F\left(x_{1}, \ldots, x_{n}\right)$ atteint ces valeurs.

Terminons ce paragraphe par une dernière remarque. Pour chaque $i=1, \ldots, m$, soit $M_{i}$ le maximum de $f_{i}$ conditionné par (1). S'il existe un point $\left(x_{1}^{*}, \ldots, x_{n}^{*}\right) \in C$ tel que $f_{i}\left(x_{1}^{*}, \ldots, x_{n}^{*}\right)=M_{i}$ pour 
chaque $i=1, \ldots, m$, alors le vecteur $\left(M_{1}, \ldots, M_{m}\right)$ (avec tous les points $\left(x_{1}, \ldots, x_{n}\right)$ où la fonction vectorielle $F \mid C$ prend cette valeur) constitue la seule solution du problème, quelle que soit l'approche adoptée. Voir paragraphe 3 , exemple 2.

\section{§2. CAS DE LA PROgRAMMATION BIVALENTE}

On appelle fonction pseudo-booléenne [6] toute fonction à valeurs réelles dont les variables prennent seulement les valeurs 0 et 1 . Dans ce paragraphe nous nous occuperons $d u$ cas où les fonctions économiques $f_{1}, \ldots, f_{m}$ sont pseudo-booléennes et les contraintes $C\left(x_{1}, \ldots, x_{n}\right)$ consistent en des équations, inégalités et mêmes conditions logiques faisant intervenir des fonctions pseudo-booléennes.

Rappelons d'abord les traits essentiels des méthodes suggérées dans [6] où nous avons traité le cas $m:=1$. Un algorithme booléen que nous appelons algorithme fondamental - basé sur un principe de programmation dynamique, nous permet de déterminer tous les points où une fonction pseudo-booléenne $f\left(x_{1}, \ldots, x_{n}\right)$ à variables indépendantes, atteint son maximum. D'autre part, tout système (1) de contraintes pseudo-booléennes peut être ramené à une seule équation booléenne $\Psi\left(x_{1}, \ldots, x_{n}\right)=1$, appelée équation caractéristique du système (1). La fonction $\Psi\left(x_{1}, \ldots, x_{n}\right)$, nommée fonction caractéristique du système (1), est mise sous forme disjonctive; à chaque conjonction (1)

$$
x_{j_{1}}^{\alpha_{j_{1}}} x_{j_{2}}^{\alpha_{j_{2}}} \ldots x_{j_{p}}^{\alpha_{\eta_{p}}}
$$

de $\Psi$, où $\alpha_{j_{1}}, \alpha_{j_{2}}, \ldots, \alpha_{j_{p}}$ sont 0 et 1 , avec la notation $x^{1}=x, x^{0}=\bar{x}$, correspond une famille (ou hyperplan) de solutions

$$
x_{j_{1}}=\alpha_{j_{1}}, \ldots, x_{j_{p}}=\alpha_{j_{p}} \text {, les autres } x_{j} \text { arbitraires }(0,1),
$$

de l'équation $\Psi=1$, donc du système (1). On obtient ainsi toutes les solutions du système pseudo-booléen (1); de plus, on peut facilement s'arranger pour que les familles de solutions soient deux-à-deux disjointes.

Il y a plusieurs possibilités pour maximiser une fonction pseudobooléenne $f\left(x_{1}, \ldots, x_{n}\right)$ sous un système pseudo-booléen (1) de restrictions. I) On peut appliquer l'algorithme fondamental à chaque famille de solutions séparément, pour choisir ensuite la plus grande valeur parmi les maxima partiels. Cette procédure peut être sensiblement accélérée, dans le cas où la fonction économique et les contraintes sont linéaires. II) On peut appliquer une adaptation de l'algorithme fondamental au cas où il y a des restrictions qu'on a ramenées préalablement à l'équation caractéristique $\Psi=1$. III) On peut appliquer l'algorithme fondamental à une nouvelle fonction pseudo-booléenne dont les variables sont indépendantes et qu'on obtient de la fonction originaire $f$ en ajoutant les fonctions qui expriment les contraintes multipliées par des constantes convenables.

(1) Les opérations booléennes sont notées par: $U$ (disjonction), ou juxtaposition (conjonction), - (négation). 
Chacun des trois procédés ci-dessus nous permet d'obtenir tous les points où la fonction $f$ atteint son maximum conditionné par (1).

Passons maintenant au cas où il y a $m$ fonctions économiques (2) pseudo-booléennes et voyons ce que devient chacune des trois approches du paragraphe précédent. Dans ce qui suit nous supposons que le système (1) de contraintes est compatible.

Approche $1^{\circ}$. Nous ne nous occuperons pas ici du problème du choix de la fonction $g$, question dont nous n'envisageons pas pour le moment une solution générale. Une fois la fonction $g$ choisie, nous obtenons un programme pseudo-booléen habituel : maximiser (3) sous les contraintes (1).

Approche $2^{\circ}$. Nous devons résoudre le problème suivant. Déterminer l'ensemble $E_{1}$ des points $x=\left(x_{1}, \ldots, x_{n}\right)$ où la fonction $f_{1}$ atteint la valeur

$$
\max _{x \in C} f_{1}(x),
$$

ensuite l'ensemble $E_{2}$ des points $x$ où la fonction $f_{2}$ prend la valeur

$$
\max _{x \in E_{1}} f_{2}(x) \text {, }
$$

ensuite l'ensemble $E_{3}$ des points $x$ qui réalisent

$$
\max _{x \in E_{2}} f_{3}(x) \text {, }
$$

etc. Or, pour résoudre le problème (23.1) on peut adopter l'une des méthodes II ou III rappelées au début de ce paragraphe; il en résultera une représentation paramétrique

$$
x=\Psi(p)
$$

(notation vectorielle pour $x_{j}=\Psi_{j}\left(p_{1}, \ldots, p_{r}\right)$ où l'on a, en général, $r<n$ ) de l'ensemble $E_{1}$. Le problème (23.2) se réduit au problème de maximum non conditionné

$$
\max _{p} f_{2}(\Psi(p))
$$

l'algorithme fondamental nous fournira donc une représentation paramétrique

$$
p=\chi(q)
$$

de l'ensemble $E_{2}$ (avec $q=\left(q_{1}, \ldots, q_{s}\right.$ ) et, en général, $s<r$ ). Le problème (23.3) se réduit maintenant au problème de maximum non conditionné

$$
\max _{q} f_{3}(\chi(q)) ;
$$

etc. Le processus sera terminé au moment où l'on aboutira à un ensemble $E_{u}$ ayant un seul élément, ou bien lorsque $u=n$. En substi- 
tuant (24.u) dans $(24 . u-1)$, celui-ci dans $(24 . u-2), \ldots$, jusqu'à (24.1), on obtiendra le(s) point(s) de l'ensemble $E_{u}$, c'est-à-dire la (les) solution(s) de notre problème.

Notons d'ailleurs qu'on peut espérer que l'ensemble $E_{1}$ contient peu d'éléments. Nous pouvons donc déterminer $E_{1}$ par la méthode I (résoudre les contraintes et maximiser séparément dans chaque famille de solutions, ce qui ne nous fournit pas une représentation paramétrique de $E_{1}$ ) et résoudre ensuite (23.2) par inspection directe de tous les points de $E_{1}$; etc.

Il y a aussi une autre méthode pour résoudre notre problème. La fonction économique $F\left(x_{1}, \ldots, x_{n}\right)$ prend ses valeurs dans l'ensemble $R^{m}$, qui a une structure d'algèbre totalement ordonnée, avec élément unité. D'autre part, le système (1) de contraintes pseudo-booléennes peut être remplacé par l'équation caractéristique $\Psi=1$. Il s'ensuit des remarques faites dans [8] que le problème de maximiser $F$ sous les contraintes (1) peut être résolu à l'aide de l'extension de l'algorithme fondamental de [6].

Approche $3^{\circ}$. Le sous-ensemble $C$ de $\{0,1\}^{n}$ étant fini, il en est de même pour $F(C)$, de sorte que nous nous trouvons bien dans les hypothèses du paragraphe $1,3^{\circ}$ et nous pouvons soit déterminer un élément maximal de $F(C)$ en appliquant la procédure $2^{\circ}$ ci-dessus, soit trouver un élément maximal de $F(C)$ qui améliore une valeur donnée de $F \mid C$.

Bien plus, nous sommes en mesure de déterminer tous les éléments maximaux de $F(C)$. En effet, soit $\Psi\left(x_{1}, \ldots, x_{n}\right)=1$ l'équation caractéristique du système $C$ des contraintes. Nous avons vu qu'en appliquant la procédure de l'approche $2^{\circ}$ on trouve un élément maximal de $F(C)$, soit $\left(y_{1}^{*}, \ldots, y_{m}^{*}\right)=F\left(x_{1}^{*}, \ldots, x_{n}^{*}\right)$. Nous devons maintenant éliminer les vecteurs $\left(x_{1}, \ldots, x_{n}\right)$ qui satisfont aux contraintes (1) mais pour lesquels on a $F\left(x_{1}, . ., x_{n}\right) \leqslant F\left(x_{1}^{*}, \ldots, x_{n}^{*}\right)$, c'est-à-dire

$$
f_{i}\left(x_{1}, \ldots, x_{n}\right) \leqslant y_{i}^{*} \quad(i=1, \ldots, m) .
$$

Par conséquent, nous allons déterminer l'équation caractéristique $\chi_{1}\left(x_{1}, \ldots, x_{n}\right)=1 \mathrm{du}$ système $(25.1)$, puis nous formerons l'équation booléenne

$$
\Psi\left(x_{1}, \ldots, x_{n}\right) \bar{\chi}_{1}\left(x_{1}, \ldots, x_{n}\right)=1 .
$$

Ensuite nous appliquerons de nouveau la procédure $2^{\circ}$ pour trouver une valeur maximale de $F$ sous la restriction (26.1), soit

$$
\left(y_{1}^{* *}, \ldots, y_{m}^{* *}\right)=F\left(x_{1}^{* *}, \ldots, x_{n}^{* *}\right) ;
$$

on voit immédiatement que $\left(y_{1}^{* *}, \ldots, y_{m}^{* *}\right)$ est un élément maximal de $F(C)$, incomparable avec $\left(y_{1}^{*}, \ldots, y_{m}^{*}\right) \cdot\left\{\right.$ Soit $\left(x_{1}, \ldots, x_{n}\right)$ satisfaisant aux contraintes $(1)$; si $\left(x_{1}, \ldots, x_{n}\right)$ satisfait $(26.1)$, alors $F\left(x_{1}, \ldots, x_{n}\right)$ ne peut majorer strictement $F\left(x_{1}^{* *}, \ldots, x_{n}^{* *}\right)$, à cause de la maximalité ; sinon, on 
a $F\left(x_{1}, \ldots, x_{n}\right) \leqslant F\left(x_{1}^{*}, \ldots, x_{n}^{*}\right)$ et la relation $F\left(x_{1}^{* *}, \ldots, x_{n}^{* *}\right) \leqslant F\left(x_{1}, \ldots, x_{n}\right)$ entraînerait $F\left(x_{1}^{* *}, \ldots, x_{n}^{* *}\right) \leqslant F\left(x_{1}^{*}, \ldots, x_{n}^{*}\right)$, donc $\chi_{1}\left(x_{1}^{* *}, \ldots, x_{n}^{* * *}\right)=1$, une contradiction. $\}$ Nous écrirons le système

$$
f_{i}\left(x_{1}, \ldots, x_{n}\right) \leqslant y_{i}^{* *} \quad(i=1, \ldots, m),
$$

dont nous trouverons l'équation caractéristique $\chi_{2}\left(x_{1}, \ldots, x_{n}\right)=1$, après quoi nous formerons l'équation

$$
\Psi\left(x_{1}, \ldots, x_{n}\right) \bar{\chi}_{1}\left(x_{1}, \ldots, x_{n}\right) \bar{\chi}_{2}\left(x_{1}, \ldots, x_{n}\right)=1,
$$

etc. Après un nombre fini de pas conduisant à des éléments maximaux de $F(C)$, (démonstration par induction), nous aboutirons à une équation

$$
\Psi\left(x_{1}, \ldots, x_{n}\right) \bar{\chi}_{1}\left(x_{1}, \ldots, x_{n}\right) \ldots \bar{\chi}_{k}\left(x_{1}, \ldots, x_{n}\right)=1
$$

incompatible. Cela signifie que

$$
\Psi\left(x_{1}, \ldots, x_{n}\right)=1 \Rightarrow \chi_{1}\left(x_{1}, \ldots, x_{n}\right) \cup \ldots \cup \chi_{k}\left(x_{1}, \ldots, x_{n}\right)=1,
$$

autrement dit toute solution $\left(x_{1}, \ldots, x_{n}\right)$ du système des contraintes satisfait au moins l'un des systèmes (25.1), (25.2), ..., (25.k), donc $F\left(x_{1}, \ldots, x_{n}\right)$ est majoré par au moins l'un des $k$ éléments maximaux trouvés. Cela montre que nous avons bien déterminé tous les éléments maximaux de $F(C)$.

La procédure que nous venons de décrire s'applique aussi au cas où il n'y a pas de contraintes $(\Psi \equiv 1)$. Cela suggère la variante suivante pour traiter le cas général. Pour chaque famille de solutions du système des contraintes, introduire les variables fixes dans les fonctions économiques, considérer les fonctions qui en résultent comme des fonctions économiques à variables indépendantes, et leur appliquer la procédure ci-dessus. Les vecteurs "provisoires" obtenus en procédant ainsi avec chaque famille de solutions ne sont pas tous des éléments maximaux de $F(C)$, mais ces derniers se trouvent nécessairement parmi les premiers. Il faudra donc finalement faire une inspection de tous les vecteurs " provisoires" et éliminer tout vecteur $\left(y_{1}, \ldots, y_{m}\right)$ qui est strictement majoré par un autre: $\left(y_{1}, \ldots, y_{m}\right)<\left(z_{1}, \ldots, z_{m}\right)$. Les vecteurs restants seront tous les éléments maximaux de $F(C)$.

Nous croyons que cette variante est efficace dans bien des cas.

\section{§ 3. EXEMPLES}

Chacun des exemples ci-dessous est destiné à illustrer plusieurs des procédures proposées aux paragraphes précédents. Nous nous abstiendrons de faire toute remarque relevant de la simplicité des exemples choisis ; nous nous bornerons plutôt à appliquer les algorithmes généraux. Les calculs intermédiaires correspondant aux sous-problèmes du type déjà étudié dans [6] (bifurcations, obtention de l'équation caractéristique, maximisation d'une seule fonction pseudo-booléenne sous contraintes données) seront simplement esquissées ou même omis. 
Exemple 1. Il y a $n$ projets pour développer une certaine activité productrice. Chaque projet $j(j=1, \ldots, n)$ assure un bénéfice annuel $b_{j}$ et une production annuelle $p_{j}$, nécessite un investissement $c_{j}$, et fournit une quantité annuelle $d_{j}$ de déchet qui sert de matière première à une autre industrie. On peut accepter plusieurs projets à la fois (par exemple en choisissant des emplacements différents pour l'application des divers projets), à la seule condition de ne pas dépasser l'investissement total $c$, mais en assurant une production annuelle totale d'au moins $p$ unités. Quels projets faut-il accepter, de façon à optimiser l'activité considérée, tout en respectant les restrictions imposées?

On voit que l'énoncé ne précise pas le sens du terme "optimiser"; ce sera à nous de le faire, en adoptant l'une des approches étudiées aux paragraphes précédents.

Introduisons les variables $x_{1}, \ldots, x_{n}$ :

$$
x_{j}=\left\{\begin{array}{l}
1, \text { si le projet } j \text { est accepté } ; \\
0, \text { dans le cas contraire } ; \quad(j=1, \ldots, n) .
\end{array}\right.
$$

On aura donc trois fonctions économiques pseudo-booléennes à maximiser :

$$
f_{1}=\sum_{j=1}^{n} b_{j} x_{j}, \quad f_{2}=-\sum_{j=1}^{n} c_{j} x_{j}, \quad f_{3}=\sum_{j=1}^{n} d_{j} x_{j}
$$

sous les restrictions

$$
\begin{aligned}
& \sum_{j=1}^{n} p_{j} x_{j} \geqslant p, \\
& \sum_{j=1}^{n} c_{j} x_{j} \leqslant c ;
\end{aligned}
$$

c'est un problème du type que nous venons d'étudier.

Considérons un exemple numérique pour $n:=6$.

\begin{tabular}{|c|cccccc|c|}
\hline$j$ & 1 & 2 & 3 & 4 & 5 & 6 & Total \\
\hline$b_{j}$ & 1 & 1 & 5 & 2 & 3 & 5 & - \\
$c_{j}$ & 7 & 6 & 4 & 4 & 2 & 1 & $c:=12$ \\
$d_{j}$ & 3 & 4 & 9 & 1 & 3 & 2 & - \\
$p_{j}$ & 2 & 3 & 2 & 5 & 8 & 7 & $p:=15$ \\
\hline
\end{tabular}

TABLEAU 1 
Nous avons les fonctions économiques :

$$
\left\{\begin{array}{l}
f_{1}=5 x_{3}+5 x_{6}+3 x_{5}+2 x_{4}+x_{1}+x_{2}, \\
f_{2}=-7 x_{1}-6 x_{2}-4 x_{3}-4 x_{4}-2 x_{5}-x_{6}, \\
f_{3}=9 x_{3}+4 x_{2}+3 x_{1}+3 x_{5}+2 x_{6}+x_{4}
\end{array}\right.
$$

et les contraintes :

$$
8 x_{5}+7 x_{6}+5 x_{4}+3 x_{2}+2 x_{1}+2 x_{3} \geqslant 15,
$$

et $\Sigma_{c_{j}} x_{j} \leqslant 12$, soit, en multipliant par -1 et en posant

$$
\begin{gathered}
-c_{j} x_{j}=c_{j} \bar{x}_{j}-c_{j} \quad(j=1, \ldots, n), \\
7 \bar{x}_{1}+6 \bar{x}_{2}+4 \bar{x}_{3}+4 \bar{x}_{4}+2 \bar{x}_{5}+\bar{x}_{6} \geqslant 12 .
\end{gathered}
$$

Comme nous l'avons dit, nous ne nous occuperons pas dans ce travail de l'approche $1^{\circ}$.

L'approche $2^{\circ}$ exige le choix d'un ordre préférentiel des fonctions économiques. Nous adoptons l'ordre $f_{1}, f_{2}, f_{3}$; autrement dit, il faut maximiser

$$
F\left(x_{1}, \ldots, x_{6}\right)=\left(f_{1}\left(x_{1}, \ldots, x_{6}\right), f_{2}\left(x_{1}, \ldots, x_{6}\right), f_{3}\left(x_{1}, \ldots, x_{6}\right)\right),
$$

par rapport à l'ordre lexicographique, sous les restrictions (32). Nous devons donc commencer par maximiser $f_{1}$ sous les restrictions (32). Appliquons l'algorithme accéléré de [6], chap. V, § 2 ; nous sommes amenés aux bifurcations successives $x_{5}:=1, x_{6}:=1, \bar{x}_{1}:=1, \bar{x}_{2}:=1$ qui donnent une première famille de solutions du système (32). Le maximum de $t_{1}$ relatif à cette famille est obtenu en posant $x_{3}:=1, x_{4}:=1$; il vient $f_{1}(0,0,1,1,1,1)=15$. Revenons aux bifurcations. Pour $x_{2}:=1$; il résulte $x_{3}:=0, x_{4}:=0$, donc une valeur plus petite pour $f_{1}$; il en est de même pour $x_{1}:=1$, qui implique $x_{2}:=0, x_{3}:=0, x_{4}:=0$. Le test accélérateur de [6] nous montre que les bifurcations $x_{6}:=0$ et $x_{5}:=0$ sont à rejeter. Nous avons donc déterminé le maximum de $f_{1}$ conditionné par (32). Toutes les variables étant fixes, nous avons trouvé la solution

$$
F(0,0,1,1,1,1)=(15,-11,15) .
$$

Passons à l'approche $3^{0}$, c'est-à-dire maximisons le vecteur (33) par rapport à l'ordre défini composante par composante, sous les restrictions (32). Comme nous l'avons observé au paragraphe 1, la solution (34.1) de l'approche $2^{\circ}$ est aussi une solution de l'approche $3^{\circ}$, c'est-à-dire un élément maximal de $F(C)$.

Proposons-nous maintenant de trouver tous les éléments de $F(C)$.

En appliquant les méthodes de [6], nous déterminons d'abord l'équation caractéristique du système $(32)$ :

$$
\begin{aligned}
\Psi=\bar{x}_{1} \bar{x}_{2} x_{5} x_{6} & \cup \bar{x}_{1} x_{2} \bar{x}_{3} \bar{x}_{4} x_{5} x_{6} \cup x_{1} \bar{x}_{2} \bar{x}_{3} \bar{x}_{4} x_{5} x_{6} \cup \\
& \cup \bar{x}_{1} x_{2} \bar{x}_{3} x_{4} x_{5} \bar{x}_{6} \cup \bar{x}_{1} \bar{x}_{2} x_{3} x_{4} x_{5} \bar{x}_{6} \cup \bar{x}_{1} x_{2} \bar{x}_{3} x_{4} \bar{x}_{5} x_{6}=1 .
\end{aligned}
$$


La première variante a pour point de départ l'obtention d'un élément maximal de $F$ sous la restriction (35). En procédant comme ci-dessus, nous trouvons le vecteur (34.1). Il faut maintenant écrire le système $\left\{f_{1} \leqslant 15, f_{2} \leqslant-11, f_{3} \leqslant 15\right\}$, ou encore

$$
\left\{\begin{array}{l}
5 \bar{x}_{3}+5 \bar{x}_{6}+3 \bar{x}_{5}+2 \bar{x}_{4}+\bar{x}_{1}+\bar{x}_{2} \geqslant 2, \\
7 x_{1}+6 x_{2}+4 x_{3}+4 x_{4}+2 x_{5}+x_{6} \geqslant 11, \\
9 \bar{x}_{3}+4 \bar{x}_{2}+3 \bar{x}_{1}+3 \bar{x}_{5}+2 \bar{x}_{6}+\bar{x}_{4} \geqslant 7 .
\end{array}\right.
$$

Nous calculons la fonction caractéristique du système (36.1):

$$
\begin{aligned}
& \chi_{1}=x_{1} x_{2} \bar{x}_{3} \cup x_{1} \bar{x}_{2} x_{3}\left(\bar{x}_{5} \cup \bar{x}_{4} \bar{x}_{6}\right) \cup x_{1} \bar{x}_{2} \bar{x}_{3} x_{4} \cup \\
& \cup \bar{x}_{1} x_{2} x_{3} \bar{x}_{5}\left(x_{4} \bar{x}_{6} \cup \bar{x}_{4} x_{6}\right) \cup \bar{x}_{1} x_{2} \bar{x}_{3} x_{4}\left(x_{5} \cup x_{6}\right) \cup \\
& \quad \cup \bar{x}_{1} \bar{x}_{2} x_{3} x_{4} x_{5} x_{6},
\end{aligned}
$$

d'où il vient

$$
\begin{aligned}
& \bar{\chi}_{1}=x_{1} x_{2} x_{3} \cup x_{1} \bar{x}_{2} x_{3} x_{5}\left(x_{4} \cup x_{6}\right) \cup x_{1} \bar{x}_{2} \bar{x}_{3} \bar{x}_{4} \cup \\
& \quad \cup \bar{x}_{1} x_{2} x_{3}\left(x_{5} \cup \bar{x}_{4} \bar{x}_{6} \cup x_{4} x_{6}\right) \cup \bar{x}_{1} x_{2} \bar{x}_{3}\left(\bar{x}_{4} \cup \bar{x}_{5} \bar{x}_{6}\right) \cup \\
& \quad \cup \bar{x}_{1} \bar{x}_{2} x_{3}\left(\bar{x}_{4} \cup \bar{x}_{5} \cup \bar{x}_{6}\right) \cup \bar{x}_{1} \bar{x}_{2} \bar{x}_{3} .
\end{aligned}
$$

Nous écrivons maintenant l'équation

$$
\begin{gathered}
\Psi \bar{\chi}_{1}=\bar{x}_{1} \bar{x}_{2} x_{3} \bar{x}_{4} x_{5} x_{6} \cup \bar{x}_{1} \bar{x}_{2} \bar{x}_{3} x_{5} x_{6} \cup \bar{x}_{1} x_{2} \bar{x}_{3} \bar{x}_{4} x_{5} x_{6} \cup \\
\cup x_{1} \bar{x}_{2} \bar{x}_{3} \bar{x}_{4} x_{5} x_{6} \cup \bar{x}_{1} \bar{x}_{2} x_{3} x_{4} x_{5} \bar{x}_{6}=1
\end{gathered}
$$

et nous devons trouver une valeur maximale de $F$ sous la restriction (37.1). A cet effet, nous appliquons de nouveau la procédure $2^{\circ}$ et commençons par maximiser $f_{1}$ sous la contrainte (37.1). On trouve facilement que ce maximum est atteint pour $\left(x_{1}, \ldots, x_{6}\right):=:(0,0,1,0,1,1)$; comme toutes les variables sont fixes, nous avons bien obtenu une valeur maximale de $F$ sous la restriction (37.1) donc (voir $\S 3,3^{\circ}$ )

$$
F(0,0,1,0,1,1)=(13,-7,14)
$$

est une valeur maximale de $F$ sous la contrainte (35). Nous écrivons maintenant le système $\left\{f_{1} \leqslant 13, f_{2} \leqslant-7, f_{3} \leqslant 14\right\}$, ou encore

$$
\left\{\begin{array}{l}
5 \bar{x}_{3}+5 \bar{x}_{6}+3 \bar{x}_{5}+2 \bar{x}_{4}+\bar{x}_{1}+\bar{x}_{2} \geqslant 4, \\
7 x_{1}+6 x_{2}+4 x_{3}+4 x_{4}+2 x_{5}+x_{6} \geqslant 7, \\
9 \bar{x}_{3}+4 \bar{x}_{2}+3 \bar{x}_{1}+3 \bar{x}_{5}+2 \bar{x}_{6}+\bar{x}_{4} \geqslant 8 .
\end{array}\right.
$$

Calculons la fonction caractéristique du système (36.2) :

$$
\begin{aligned}
\chi_{2}= & \bar{x}_{1} x_{2} x_{3} \bar{x}_{5} \bar{x}_{6} \cup x_{1} \bar{x}_{2} x_{3} \bar{x}_{5}\left(\bar{x}_{4} \cup \bar{x}_{6}\right) \cup \\
& \cup \bar{x}_{1} \bar{x}_{2} x_{3}\left(x_{4}\left(\bar{x}_{5} \cup \bar{x}_{6}\right) \cup \bar{x}_{4} x_{5} x_{6}\right) \cup \bar{x}_{1} x_{2} \bar{x}_{3}\left(x_{4} \cup x_{5} \cup x_{6}\right) \cup \\
& \cup \bar{x}_{1} \bar{x}_{2} \bar{x}_{3} x_{4} x_{5} x_{6} \cup x_{1} x_{2} \bar{x}_{3} \cup \bar{x}_{1} \bar{x}_{2} x_{3},
\end{aligned}
$$


d'où il vient

$$
\begin{aligned}
\bar{\chi}_{2} & =\bar{x}_{1} x_{2} x_{3}\left(x_{5} \cup x_{6}\right) \cup x_{1} \bar{x}_{2} x_{3}\left(x_{5} \cup x_{4} x_{6}\right) \cup \\
& \cup \bar{x}_{1} \bar{x}_{2} x_{3}\left(x_{4} x_{5} x_{6} \cup \bar{x}_{4}\left(\bar{x}_{5} \cup \bar{x}_{6}\right)\right) \cup \bar{x}_{1} x_{2} \bar{x}_{3} \bar{x}_{4} \bar{x}_{5} \bar{x}_{6} \cup \\
& \cup \bar{x}_{1} \bar{x}_{2} \bar{x}_{3}\left(\bar{x}_{4} \cup \bar{x}_{5} \cup \bar{x}_{6}\right) \cup x_{1} x_{2} x_{3} .
\end{aligned}
$$

Nous écrivons maintenant l'équation

$$
\Psi \bar{\chi}_{1} \bar{\chi}_{2}=\bar{x}_{1} \bar{x}_{2} \bar{x}_{3} \bar{x}_{4} x_{5} x_{6}=1
$$

et nous devons trouver une valeur maximale de $F$ sous la restriction (37.2). Cette dernière équation n'ayant qu'une seule solution, l'unique valeur

$$
F(0,0,0,01,1)=(8,-3,5)
$$

est forcément maximale sous la restriction (37.2), donc maximale sous la restriction (35). La fonction caractéristique $\chi_{3}$ du système

$$
\left\{f_{1} \leqslant 8, f_{2} \leqslant-3, f_{3} \leqslant 5\right\}
$$

satisfait l'inégalité $\chi_{3} \geqslant \bar{x}_{1} \bar{x}_{2} \bar{x}_{3} \bar{x}_{4} x_{5} x_{6}$, d'où il résulte que l'équation

$$
\Psi \bar{\chi}_{1} \bar{\chi}_{2} \bar{\chi}_{3}=1
$$

n'a pas des solutions. Il s'ensuit que (34.1), (34.2) et (34.3) sont les seules valeurs maximales de $F$ sous les restrictions (32).

La deuxième variante pour déterminer les éléments maximaux de $F(C)$ consiste en l'application de la première variante à chaque famille de solutions du système de contraintes, suivie de l'élimination des valeurs qui ne sont pas maximales. Les familles de solutions du système (32) correspondent aux disjonctions de l'équation caractéristique (35).

La première famille de solutions $\left(x_{1}:=x_{2}:=0, x_{5}:=x_{6}:=1\right.$, $x_{3}, x_{4}$ arbitraires), réduit les fonctions économiques (31) aux fonctions suivantes :

$\left(31^{\prime}\right) f_{1}^{\prime}=5 x_{3}+2 x_{4}+8, \quad f_{2}^{\prime}=-4 x_{3}-4 x_{4}-3, \quad f_{3}^{\prime}=9 x_{3}+x_{4}+5 ;$

il faut maintenant déterminer les valeurs maximales du vecteur

$$
F^{\prime}\left(x_{3}, x_{4}\right)=\left(f_{1}^{\prime}\left(x_{3}, x_{4}\right), f_{2}^{\prime}\left(x_{3}, x_{4}\right), f_{3}^{\prime}\left(x_{3}, x_{4}\right)\right) \text {. }
$$

Le maximum de $f_{1}^{\prime}$ est obtenu pour $x_{3}:=1, x_{4}:=1$; toutes les variables étant fixes, nous avons obtenu la valeur maximale

$$
F^{\prime}(1,1)=(15,-11,15) \text {. }
$$

Le système $\left\{f_{1}^{\prime} \leqslant 15, f_{2}^{\prime} \leqslant-11, f_{3}^{\prime} \leqslant 15\right\}$ s'écrit

$$
\left\{5 \bar{x}_{3}+2 \bar{x}_{4} \geqslant 0,4 x_{3}+4 x_{4} \geqslant 8,9 \bar{x}_{3}+\bar{x}_{4} \geqslant 0\right\} ;
$$

on voit immédiatement que sa fonction caractéristique est $\chi_{1}^{\prime},=x_{3} x_{4}$. Nous devons maintenant maximiser $F^{\prime}$ sous la contrainte $\bar{\chi}_{1}^{\prime}=\bar{x}_{3} \cup \bar{x}_{4}=1$. 
On obtient le maximum conditionné de $f_{1}^{\prime}$ pour $x_{3}:=1, x_{4}:=0$; toutes les variables étant fixes, nous avons trouvé la valeur maximale $F^{\prime}(1,0)=(13,-7,14)$. Le système $\left\{f_{1}^{\prime} \leqslant 13, f_{2}^{\prime} \leqslant-7, f_{3}^{\prime} \leqslant 14\right\}$ s'écrit $\left\{5 \bar{x}_{3}+2 \bar{x}_{4} \geqslant 2,4 x_{3}+4 x_{4} \geqslant 4,9 \bar{x}_{3}+\bar{x}_{4} \geqslant 1\right\}$; on voit immédiatement que sa fonction caractéristique $\chi_{2}^{\prime}=x_{3} \bar{x}_{4} \cup \bar{x}_{3} x_{4}$. Nous devons maintenant maximiser $F^{\prime}$ sous la contrainte $\bar{\chi}_{1}^{\prime} \bar{\chi}_{2}^{\prime}=\bar{x}_{3} \bar{x}_{4}=1$. Cette équation a l'unique solution $x_{3}:=0, x_{4}:=0$, d'où il vient la valeur maximale $F^{\prime}(0,0)=(8,-3,5)$ et $\chi_{3}^{\prime} \geqslant \bar{x}_{3} \bar{x}_{4}$, donc l'équation $\bar{\chi}_{1}^{\prime} \bar{\chi}_{2}^{\prime} \bar{\chi}_{3}^{\prime}=1$ est incompatible.

Les autres familles de solutions étant dégénérées (chacune est réduite à une seule solution), l'unique valeur de $F$ qui correspond à chaque famille est forcément maximale dans cette famille-là. En résumant, nous obtenons le tableau 2 (p. 28).

Dernière étape : élimination des valeurs non maximales. Les valeurs $(9,-9,9),(9,-10,8),(6,-12,8),(10,-10,13)$ et $(8,-11,7)$ ne sont pas maximales, car elles sont majorées par $(13,-7,14)$. Les trois valeurs correspondant à la première famille de solutions sont deux-à-deux incomparables, d'où il résulte qu'elles sont maximales. Nous avons donc retrouvé le même résultat que par la première variante.

On notera que le vecteur $(13,-7,14)$ est une valeur maximale de $F$ sous les contraintes (32), bien qu'aucune de ses composantes ne soit le maximum de la fonction correspondante $f_{i}$ conditionné par (32).

Exemple 2. Maximiser les fonctions

$$
\begin{aligned}
& f_{1}=4 x_{1} x_{3} x_{4}+3 x_{1} x_{2} x_{4}-6 \bar{x}_{1} x_{3} x_{4}+5 x_{1} \bar{x}_{3} x_{4}+5 x_{1} x_{2} \bar{x}_{3} \bar{x}_{4}, \\
& f_{2}=2 \bar{x}_{3}+3 x_{4}-7 \bar{x}_{5}-5 \bar{x}_{3} x_{4} \bar{x}_{5}+3 x_{4} x_{6}+9 x_{6} \bar{x}_{7}-2 \bar{x}_{3} \bar{x}_{7}
\end{aligned}
$$

sous la condition

$$
x_{1} \bar{x}_{2} \bar{x}_{3} x_{5} \cup x_{1} \bar{x}_{3} \bar{x}_{4} \bar{x}_{6} x_{7} \cup \bar{x}_{1} x_{2} x_{3} x_{6} \bar{x}_{7} \cup \bar{x}_{2} x_{3} x_{4} \bar{x}_{5} x_{7} \cup \bar{x}_{1} x_{3} \bar{x}_{4} x_{5} \bar{x}_{6}=1 .
$$

Nous adopterons l'approche $2^{\circ}$, en choisissant l'ordre préférentiel $f_{1}, f_{2}$. Il faut donc commencer par maximiser $f_{1}$ sous la restriction (39). Nous calculons immédiatement les maxima de $f_{1}$ relatifs aux familles de solutions de (39), d'où il résulte, par simple inspection, que le maximum de $f_{1}$ conditionné par (39) est

$$
f_{1}\left(1,0,0,1,1, x_{6}, x_{7}\right)=f_{1}\left(1,1,0,0, x_{5}, 0,1\right)=5 .
$$

Nous obtenons sans peine une représentation paramétrique des points de maximum :

$$
\begin{aligned}
x_{1}:=1, x_{2}:=p, x_{3}:=0, x_{4}:=\bar{p} & \\
& x_{5}:=\bar{p} \cup q, x_{6}:=\bar{p} r, x_{7}:=p \cup s ; p, q, r, s \text { arbitraires. }
\end{aligned}
$$

En substituant (41) dans (38.1) et (39), il nous reste à maximiser $\left(38^{\prime} .2\right)$

$$
f_{2}^{\prime}=2+3 \bar{p}-7 \bar{p} \bar{q}+3 \bar{p} r+9 \bar{p} r \bar{s}-2 \bar{p} \bar{s}
$$


sous la restriction $\bar{p}(\bar{p} \cup q) \cup p(p \cup \bar{r})(p \cup s)=1$, qui est identiquement satisfaite.

Le même procédé nous montre que le maximum de $f_{2}^{\prime}$ est 15 ; il est atteint pour $p:=0, q:=1, r:=1, s:=0$. En substituant dans (41) et en posant

$$
F\left(x_{1}, \ldots, x_{7}\right)=\left(f_{1}\left(x_{1}, \ldots, x_{7}\right), f_{2}\left(x_{1}, \ldots, x_{7}\right)\right),
$$

on obtient la valeur maximum

$$
F(1,0,0,1,1,1,0)=(5,15)
$$

conditionnée par (39).

Comme nous l'avons dit au paragraphe $2,2^{\circ}$, une autre variante consiste en l'application directe de l'algorithme fondamental de maximisation à la fonction $F$ conditionnée par (39). On obtient le tableau 3 (p. 28).

Les calculs sont effectués dans l'algèbre $R^{2}$, dont les opérations sont définies par $(a, b) \pm(c, d)=(a \pm c, b \pm d),(a, b)(c, d)=(a c, b d)$, $(a, b) x=(a x, b x)$; l'algèbre est munie de l'ordre lexicographique. A titre d'exemple, reproduisons les calculs pour la maximisation dans la première famille de solutions. On a

$$
F=\left[(5,3)+(0,3) x_{6}\right] x_{4}+(0,2)+(0,9) x_{6} \bar{x}_{7}-(0,2) \bar{x}_{7} ;
$$

étant donné qu'on a toujours $(5,3)+(0,3) x_{6}>(0,0)$ (l'élément nul de $R^{2}$ ), il résulte $x_{4}:=1$, donc

$$
\begin{aligned}
F & =(5,3)+(0,2)+(0,3) x_{6}+(0,9) x_{6} \bar{x}_{7}-(0,2) \bar{x}_{7} \\
& =\left[(0,3)+(0,9) \bar{x}_{7}\right] \cdot x_{6}+(5,5)-(0,2) \bar{x}_{7} .
\end{aligned}
$$

Il en résulte de même $x_{6}:=1$, donc

$$
F=(0,3)+(0,9) \bar{x}_{7}+(5,5)-(0,2) \bar{x}_{7}=(0,7) \bar{x}_{7}+(5,8),
$$

donc $\bar{x}_{7}:=1$, car $(0,7)>(0,0)$, par conséquent la valeur maximum est $F=(0,7)+(5,8)=(5,15)$.

On procède de même avec les autres familles de solutions. La comparaison des valeurs inscrites au tableau 3 montre que $(5,15)$ est la plus grande valeur de $F$ conditionnée par (39).

Une dernière remarque. Nous avons vu que le maximum de $f_{1}$ conditionné par (39) est 5 . On peut aisément vérifier que le maximum de $f_{2}$ conditionné par (39) est 15. Par conséquent, si l'on considère l'ordre partiel défini composante par composante, c'est-à-dire si l'on adopte l'approche $3^{\circ}$, on voit que l'ense mble $F(C)$ admet l'élément $(5,15)$ comme plus grand élément. Voilà donc un exemple où il existe un élément maximum (nécessairement unique!) au lieu de plusieurs éléments maximaux. 

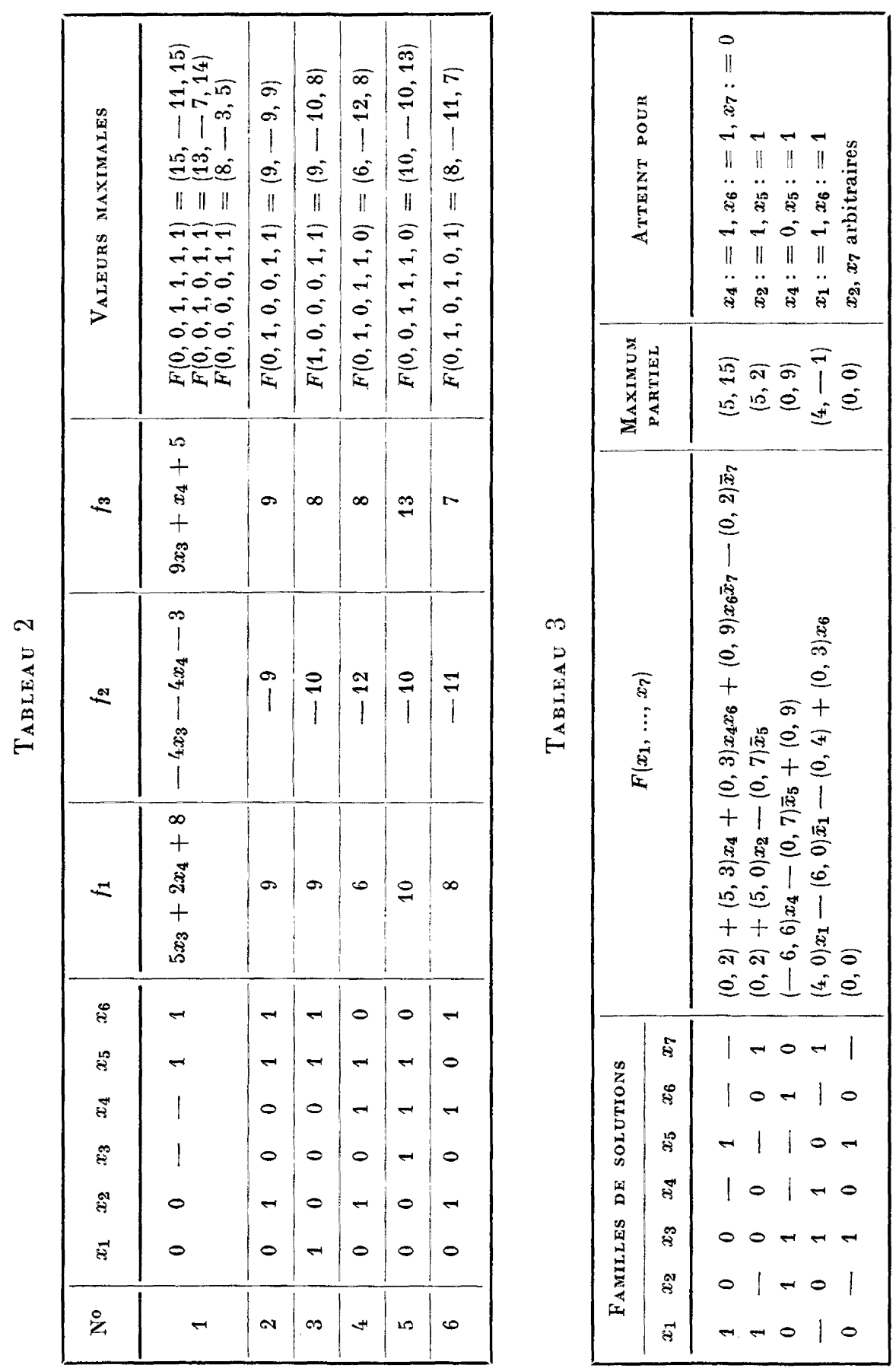


\section{§4. REMARQUES FINALES ET CONCLUSION}

On peut aussi envisager la combinaison suivante entre les approches $2^{\circ}$ et $3^{\circ}$ : partager les critères d'optimum en classes de critères équivalents, et établir un ordre préférentiel entre les diverses classes. C'est précisément le point de vue "Electre " [4], [7], qui nous fournit un guide pour établir les classes de critères équivalents et la hiérarchie entre ces classes (voir, par exemple, [4], troisième application).

Soit $F_{1}\left(x_{1}, \ldots, x_{n}\right)$ le vecteur formé par les fonctions économiques appartenant à la première classe d'équivalence. Nous trouverons toutes les valeurs maximales de $F_{1} \mid C$ en appliquant l'approche $3^{\circ}$. Soit $E_{1}$ l'ensemble des points où la fonction $F_{1}$ atteint ces valeurs, et soit $F_{2}\left(x_{1}, \ldots, x_{n}\right)$ le vecteur formé par les fonctions économiques qui appartiennent à la seconde classe d'équivalence ; il faut maintenant déterminer les valeurs maximales de $F_{2} \mid E_{1}$ et l'ensemble $E_{2}$ des points où $F_{2} \mid E_{1}$ atteint ces valeurs; etc. Il convient de noter qu'on obtient un noyau du graphe mentionné dans [4] en choisissant un et un seul point dans chacun des ensembles $E_{k}$.

Ce point de vue, plus approfondi, mérite d'être étudié de plus près dans un prochain travail.

\section{BIBLIOGRAPHIE}

[1] J. Aurian, Méthode d'optimisation de l'emplacement des entreprises. Communication présentée à la Session scientifique annuelle du Centre de calcul économique et cybernétique économique. Bucarest, 21-22 février 1969.

[2] P. Bod, Programmation linéaire dans le cas de plusieurs fonctions-objectif données simultanément (en Hongrois). Publ. Math. Inst. Hungar. Acad. Sci. (series B), 8 (1963), 541-554.

[3] Gh. Boldur, V. Ionescu et I. M. Stancu-Mrnasian, Application de la théorie de l'utilité à la résolution des problèmes de programmation linéaire à plusieurs critères d'optimum. Communication présentée à la Session scientifique annuelle du Centre de calcul économique et cybernétique économique. Bucarest, 21-22 février 1969.

[4] P. Buffet, J.-P. Gremy, M. Marc et B. Sussmann, Peut-on choisir en tenant compte de critères multiples ? Une méthode (Electre) et trois applications. Metra, 6 (1967), 283-316.

[5] A. M. Geoffrion, Solving Bicriterion Mathematical Programs. Operations Research, 15 (1967), 39-54.

[6] P. L. Hammer et S. Rudeanu, Boolean Methods in Operations Research and Related Areas. Springer-Verlag, Berlin/Heidelberg/New York, 1968.

[7] B. Roy, Classement et choix en présence de points de vue multiples (La méthode Electre.) R.I.R.O., 2 (1968), no 8, 57-75.

[8] S. Rudeanu, An Axiomatic Approach to Pseudo-Boolean Programming. 